\title{
Direct thrust measurements and modelling of a radio-frequency expanding plasma thruster
}

\author{
T. Lafleur, ${ }^{1, a)}$ K. Takahashi, ${ }^{1,2}$ C. Charles, ${ }^{1}$ and R. W. Boswell ${ }^{1}$ \\ ${ }^{1}$ Space Plasma, Power and Propulsion Group, Research School of Physics and Engineering, The Australian \\ National University, Canberra ACT 0200, Australia \\ ${ }^{2}$ Department of Electrical Engineering and Computer Science, Iwate University, Morioka 020-8551, Japan
}

(Received 22 March 2011; accepted 14 June 2011; published online 1 August 2011)

\begin{abstract}
It is shown analytically that the thrust from a simple plasma thruster (in the absence of a magnetic field) is given by the maximum upstream electron pressure, even if the plasma diverges downstream. Direct thrust measurements of a thruster are then performed using a pendulum thrust balance and a laser displacement sensor. A maximum thrust of about $2 \mathrm{mN}$ is obtained at $700 \mathrm{~W}$ for a thruster length of $17.5 \mathrm{~cm}$ and a flow rate of $0.9 \mathrm{mg} \mathrm{s}^{-1}$, while a larger thrust of $4 \mathrm{mN}$ is obtained at a similar power for a length of $9.5 \mathrm{~cm}$ and a flow rate of $1.65 \mathrm{mg} \mathrm{s}^{-1}$. The measured thrusts are in good agreement with the maximum upstream electron pressure found from measurements of the plasma parameters and in fair agreement with a simple global approach used to model the thruster. (C) 2011 American Institute of Physics. [doi:10.1063/1.3610570]
\end{abstract}

Expanding plasma and double layer (DL) thrusters ${ }^{1-4}$ are receiving increasing interest due to their potential advantages over conventional systems such as ion or hall thrusters. Such advantages include the lack of an explicit neutralizer, high power and thrust densities, and the absence of biased electrodes. ${ }^{1}$ These systems typically produce plasma within an insulating source tube through radio-frequency (rf) means and make use of a magnetic field to help confine the plasma and produce expansion at the thruster exit. ${ }^{1,2,5}$ The plasma is then accelerated by ambipolar or double layer fields that form internal to the plasma. ${ }^{1,2,4,6}$ While prolific studies of the fundamental physics in such systems have been done, detailed experimental measurements of the thrust of such devices are only now beginning to be performed. ${ }^{7,8}$ These thrust measurements are vital, as they allow almost all other electric propulsion figures of merit to be determined, ${ }^{9}$ and thus, the performance of such systems to be assessed.

Fruchtman has provided much of the theoretical groundwork for such plasma thrusters and has performed a number of detailed analyses in collisionless ${ }^{10}$ and collisional regimes, ${ }^{11}$ as well as investigating the effects of neutral depletion $^{10,11}$ and magnetic expansion. ${ }^{12}$ In particular, in the absence of a magnetic field, the thrust has been predicted to be strongly linked to the maximum upstream electron pressure. ${ }^{10}$ Recent direct thrust measurements ${ }^{7}$ of a permanent magnet helicon double layer thruster (PM-HDLT) have agreed favourably with this result; however, it is unclear how the presence of both the DL and the magnetic field affects the measured thrust. In order to test these theoretical results conclusively, the thrust measurements in a system without a magnetic field or DL are needed. With an improved understanding of the physics involved in the thrust delivery mechanism, better optimization studies can be performed.

In the present letter, we experimentally investigate the thrust of a simple rf plasma thruster in the absence of a magnetic

\footnotetext{
${ }^{\text {a)} E l e c t r o n i c ~ m a i l: ~ t r e v o r . l a f l e u r @ a n u . e d u . a u . ~}$
}

field and under conditions where a DL is not present. We also extend the theoretical results of Fruchtman ${ }^{10}$ by assessing the effect that plasma divergence has on the expected system thrust.

We begin by addressing the question of how the thrust of a plasma thruster is affected by possible plasma divergence downstream of the thruster exit. The steady state collisionless momentum equation (in the absence of a magnetic field) for a particular plasma species, $j$, is given by ${ }^{13}$

$$
M_{j} \nabla \cdot\left(n_{j} \mathbf{v}_{j} \mathbf{v}_{j}\right)=q_{j} n_{j} \mathbf{E}-\nabla p_{j}
$$

where $M_{j}, n_{j}, \mathbf{v}_{j}, q_{j}$, and $p_{j}$ are the mass, density, velocity, charge, and pressure of the particular plasma species, respectively. Here, we have assumed that the particle pressure is isotropic. By then treating an electropositive, quasi-neutral plasma with cold, singly charged ions, the ion momentum equation (using cylindrical coordinates and assuming azimuthal symmetry) in the axial $(z)$ direction is

$$
\frac{\partial}{\partial z}\left(M n v_{z}^{2}\right)+\frac{1}{r} \frac{\partial}{\partial r}\left(r M n v_{r} v_{z}\right)=q n E_{z}
$$

where $M$ is the ion mass, $n$ is the plasma density, $v_{r}$ and $v_{z}$ are the ion flow velocity components, $E_{z}$ is the axial electric field, and $q$ is the charge magnitude. The electron momentum equation (ignoring electron inertia) is

$$
0=-q n E_{z}-\frac{\partial}{\partial z}\left(q n T_{e}\right)
$$

where $T_{e}$ is the electron temperature in $\mathrm{eV}$. By adding Eqs. (2) and (3) together and rearranging, we have

$$
\frac{\partial \tau}{\partial z}=-\frac{1}{r} \frac{\partial}{\partial r}\left(r M n v_{r} v_{z}\right)
$$

where $\tau=M n v_{z}^{2}+q n T_{e}$. We now identify $\tau$ as an axial force per unit area and see that this force per unit area changes along $z$ as momentum flows into or out of the differential 
volume. We are however interested in finding the total axial force, $T$. To do this we integrate both sides of Eq. (4) in the radial $(r)$ and azimuthal $(\theta)$ directions and define

$$
T=\int_{0}^{2 \pi} \int_{0}^{R(z)} \tau(r, z) r d r d \theta
$$

where $R(z)$ is the radial integration boundary at each axial location. Thus by integrating Eq. (4), we obtain

$$
\frac{d T}{d z}=-2 \pi M\left[r n v_{r} v_{z}\right]_{r=R(z)} .
$$

Outside the thruster, by choosing $R(z)$ large enough, the plasma density will be zero or close to zero, so that the righthand side of Eq. (6) vanishes. Inside the thruster, the density is not zero at the radial boundaries, and the right-hand side of Eq. (6) represents a friction force on the radial thruster walls. By ignoring this friction force, we can also set the right-hand side to zero. Although ions are lost at the walls, these are mainly the axially slower ions, and thus, the axial momentum loss to the walls is small. Thus $d T / d z=0$, and, therefore, $\quad T=M\left\langle n v_{z}^{2}\right\rangle A_{e}+q\left\langle n T_{e}\right\rangle A_{e}=$ constant, where $\left\langle n v_{z}^{2}\right\rangle$ and $\left\langle n T_{e}\right\rangle$ are cross-section averaged quantities, and $A_{e}$ is the thruster cross-sectional area. If we further assume that the electron temperature is constant and that the ion axial velocity is zero along a single plane, then the electron pressure is a maximum when the ion flow velocity is zero, and thus,

$$
T=q\langle n\rangle_{\max } A_{e} T_{e}
$$

where $\langle n\rangle_{\max }$ is the maximum cross-section averaged plasma density. This result says that even though the plasma might diverge downstream, the total axial flow momentum must be conserved, and the thrust is still given by the maximum upstream electron pressure. We now conduct a series of measurements to confirm that the thrust of a plasma thruster is indeed given by this upstream pressure.

Experiments are performed using a simple cylindrical plasma thruster, a schematic of which is shown in Fig. 1. The thruster consists of a Pyrex source tube of diameter 6.4 $\mathrm{cm}$ open at one end and closed at the other end by a moveable insulating plate that allows the effective plasma cavity length to be adjusted. The source tube is surrounded by a copper double-turn loop antenna connected to a rf power generator (operated at $13.56 \mathrm{MHz}$ ) through a $\pi$ matching network. Argon gas is fed into the thruster via a feedthrough that passes through the insulating plate. The thruster is placed inside the larger Irukandji space simulation chamber, ${ }^{14}$ which is $1.4 \mathrm{~m}$ long and has a diameter of $1 \mathrm{~m}$. This chamber is connected to a turbomolecular/rotary pump system, and system pressures are measured with a combination of ion and baratron gauges.

The plasma thruster is mounted onto a grounded thrust balance described previously. ${ }^{7,8}$ This balance consists of 4 aluminium support columns (not shown in Fig. 1) onto which a double pendulum structure is attached. The pendulum hinges are constructed using $0.1 \mathrm{~mm}$ thick stainless steel flexible plates, and the thruster connects to the bottom of the pendulum. The pendulum displacement is measured with a

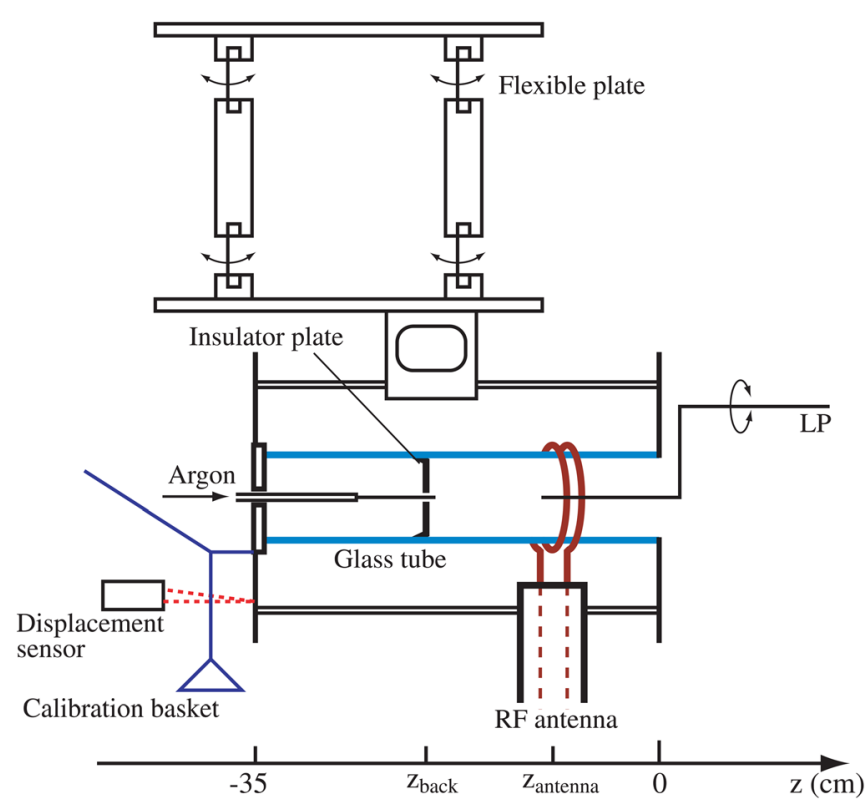

FIG. 1. (Color online) Schematic of the rf plasma thruster attached to the thrust balance. Also shown is the laser displacement sensor, thrust balance calibration system, rf antenna, and LP.

high sensitivity rf-shielded Micro-Epsilon ILD 1700 - 2 laser displacement sensor mounted to the thrust balance support structure. As in previous studies, ${ }^{7}$ both the gas feedthrough and rf antenna are not in physical contact with the thruster source tube. With the plasma off, no measurable thrust is observed with the rf on (no gas) and also with the gas on (rf off).

When taking thrust measurements, the laser sensor is initially run for $20 \mathrm{~s}$ with no plasma to obtain an equilibrium displacement level, following which the plasma is turned on and measurements taken for $5 \mathrm{~s}$. The plasma is then turned off and measurements are taken for a further $20 \mathrm{~s}$. The laser sensor data are then filtered to remove the mechanical oscillations of the pendulum $(\approx 1 \mathrm{~Hz})$, and the thrust displacement then determined. To calibrate the laser system, known masses are placed in a small calibration basket attached to the thrust balance support structure and thruster (see Fig. 1). From static force equilibrium considerations, the applied horizontal "thrust" force can be determined. The sensitivity was found to be about $230 \mathrm{mN} / \mathrm{mm}$, with a resolution of $0.023 \mathrm{mN}$. This is better than in previous studies, ${ }^{7}$ since the present system is lighter due to the absence of the permanent magnets used there. During calibration, both the gas flow and $\mathrm{rf}$ are off.

Plasma parameters (i.e., density and electron temperature) are measured with a $4 \mathrm{~mm}$ diameter disc Langmuir probe (LP). The plasma density is found from the ion saturation region of the measured $I V$ curve (obtained using a standard sweeping circuit), while the electron temperature is found from the natural logarithm of the slope of the $I V$ curve after subtracting off the ion current contribution. Sheridan's method $^{15}$ is used to account for expansion of the sheath around the LP disc.

To investigate the plasma parameters and thrust of the thruster, two test cases are used. In the first case, the cavity length is set to $9.5 \mathrm{~cm}$ (with the antenna located at $z=-5.5 \mathrm{~cm}$ ) and 
the gas flow rate is $1.65 \mathrm{mg} \mathrm{s}^{-1}$ (giving a chamber pressure of $0.24 \mathrm{~Pa}$ ), while in the second case, the cavity length is $17.5 \mathrm{~cm}$ (with the antenna located at $z=-9 \mathrm{~cm}$ ) and the gas flow rate is $0.9 \mathrm{mg} \mathrm{s}^{-1}$ (giving a chamber pressure of $0.13 \mathrm{~Pa}$ ). The measured maximum upstream plasma density and upstream electron temperature are shown in Figs. 2(a) and 2(b). The results are all plotted against effective power, which is the total power absorbed by the plasma. These powers are determined by taking resistance measurements (with an rf current probe) of the antenna/plasma system to establish the power transfer efficiency ${ }^{16}$ (measured to be between $60 \%-80 \%$ ).

Figure 2(a) shows that the plasma density for the long cavity (closed circles; taken at $z=-10 \mathrm{~cm}$ ) is approximately linear with effective power, going from around $4 \times 10^{17} \mathrm{~m}^{-3}$ at $125 \mathrm{~W}$ to around $13 \times 10^{17} \mathrm{~m}^{-3}$ at $650 \mathrm{~W}$. A similar result is seen with the short cavity (closed squares; taken at $z=-6 \mathrm{~cm}$ ), except with higher densities of $8.5 \times 10^{17} \mathrm{~m}^{-3}$ at $140 \mathrm{~W}$ and about $30 \times 10^{17} \mathrm{~m}^{-3}$ at 680 W. The measured electron temperatures in Fig. 2(b) show that for both cases the temperature is approximately constant with effective power, with a value of about $5.5 \mathrm{eV}$ for the long cavity and $4 \mathrm{eV}$ for the short cavity.

Figure 3 shows the thrust measurements from the thrust balance for the long cavity (closed circles) and short cavity
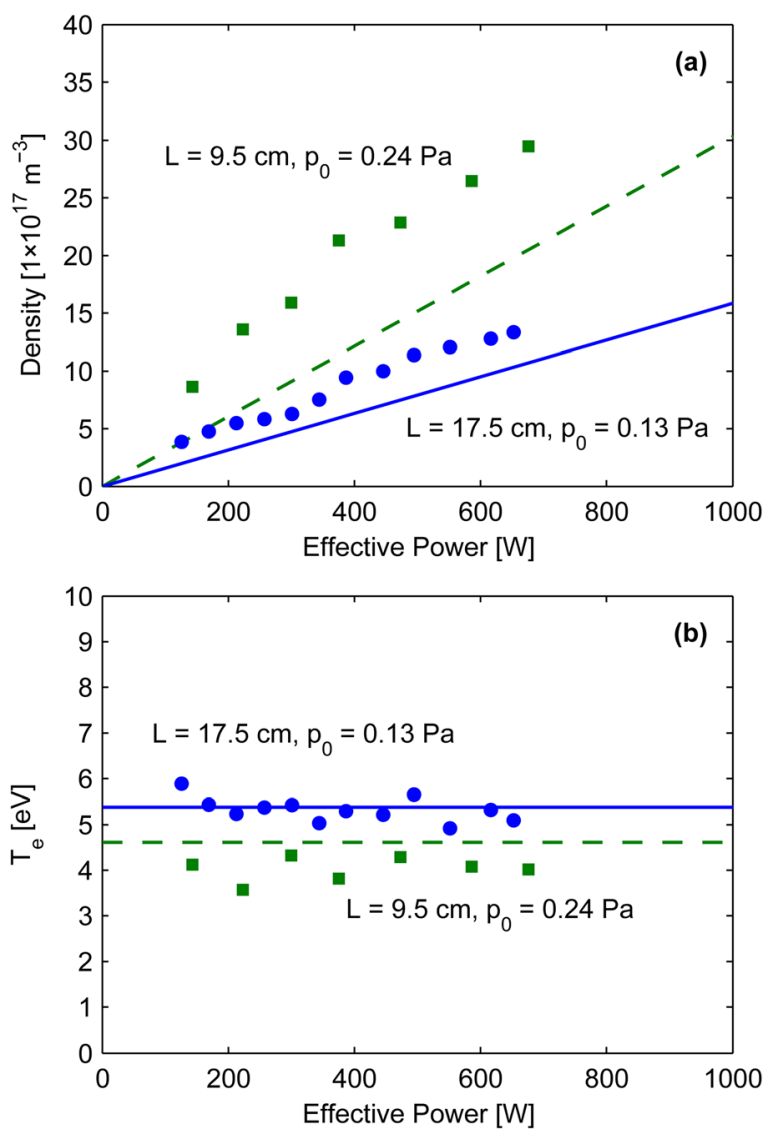

FIG. 2. (Color online) (a) Maximum upstream plasma density as a function of effective power. The closed squares show the measured results at $z=-6$ $\mathrm{cm}$ for a cavity length of $9.5 \mathrm{~cm}$ and an argon flow rate of $1.65 \mathrm{mg} \mathrm{s}^{-1}$ (giving a working pressure of $0.24 \mathrm{~Pa}$ ), while the closed circles show results at $z=-10 \mathrm{~cm}$ for a cavity length of $17.5 \mathrm{~cm}$ and an argon flow rate of $0.9 \mathrm{mg}$ $\mathrm{s}^{-1}$ (giving a working pressure of $0.13 \mathrm{~Pa}$ ). The dashed line and solid line show the global model results for each of the respective cases. (b) Upstream electron temperatures for the cases in (a).

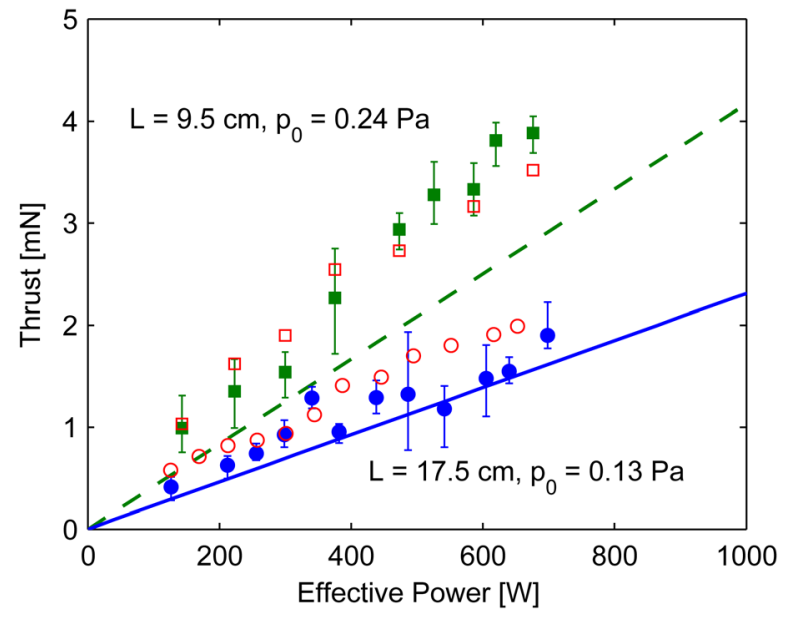

FIG. 3. (Color online) Thrust as a function of effective power. The closed squares show the measured results at $z=-6 \mathrm{~cm}$ for a cavity length of 9.5 $\mathrm{cm}$ and an argon flow rate of $1.65 \mathrm{mg} \mathrm{s}^{-1}$ (giving a working pressure of 0.24 $\mathrm{Pa})$, while the closed circles show results at $z=-10 \mathrm{~cm}$ for a cavity length of $17.5 \mathrm{~cm}$ and an argon flow rate of $0.9 \mathrm{mg} \mathrm{s}^{-1}$ (giving a working pressure of $0.13 \mathrm{~Pa}$ ). The dashed line and solid line show the global model results for each of the respective cases, while the open squares and open circles show the calculated thrust using the measured plasma parameters in Figs. 2(a) and 2(b), a scaling factor to account for the radial density profile, and Eq. (7).

(closed squares), where similarly to the densities in Fig. 2(a), the results are approximately linear with effective power. The thrust reaches a maximum of about $2 \mathrm{mN}$ at $700 \mathrm{~W}$ for the long cavity and around $4 \mathrm{mN}$ at a similar power for the short cavity. Also plotted in Fig. 3 are the calculated thrusts from Eq. (7) (open squares and open circles) using the plasma parameters obtained in Figs. 2(a) and 2(b). Equation (7) requires the cross-section averaged density, which is found by multiplying the densities in Fig. 2(a) with a scaling factor $\left(\gamma_{9.5} \approx 0.58\right.$ and $\gamma_{17.5} \approx 0.53$ for the short and long cavities, respectively) obtained from a measurement of the radial density profiles within the source region. As seen, the calculated thrusts are in good agreement with those found from the thrust balance, and since the electron temperature remains roughly constant with power (see Fig. 2(b)), this shows that the increasing thrust is a sole result of the increasing plasma density. These results show that the total thrust force is given by the maximum upstream electron pressure, which in the present system serves as the only source of momentum for the plasma. Thus, if the upstream density and electron temperature can be predicted, the thrust can be determined directly from Eq. (7) without needing to account for plasma divergence effects at the thruster exit.

Insight into the results in Figs. 2-3 can be obtained by making use of a simple global model. By considering a uniform plasma density discharge together with a constant neutral gas density and assuming that ions reach the Bohm velocity at the thruster exit, the electron temperature can be found by equating the total particle loss and generation rates, ${ }^{17}$

$$
n_{0} A u_{B}=n_{0} n_{g} K_{i z} V
$$

where $n_{0}$ is the plasma density, $A=h_{L}\left(2 \pi R^{2}\right)+h_{R}(2 \pi R L)$ is the total effective loss area with $R$ and $L$, the source tube 
radius and length, respectively, and $h_{L}$ and $h_{R}$ the ratio of the sheath-to-center density in the axial and radial directions, respectively, ${ }^{17} u_{B}=\sqrt{q T_{e} / M}$ is the Bohm velocity, $n_{g}$ is the neutral gas density, $K_{i z}=K_{i z}\left(T_{e}\right)$ is the ionization rate factor for Maxwellian electrons, and $V=\pi R^{2} L$ is the total ionization volume. The plasma density can then be found from a power balance, ${ }^{17}$

$$
P_{a b s}=q n_{0} A u_{B} E_{T},
$$

where $P_{a b s}$ is the total absorbed power, and $E_{T}=E_{c}+E_{i}$ $+E_{e}$ is the energy loss per electron-ion per lost from the system (measured in $\mathrm{eV}$ ), with $E_{c}=E_{\text {ion }}+\left(K_{\text {exc }} / K_{i z}\right) E_{\text {exc }}$ $+(3 m / M)\left(K_{e l} / K_{i z}\right) T_{e}$ the collisional energy loss and $E_{i}+E_{e}=\frac{1}{2} T_{e}+V_{s}+2 T_{e}$ the kinetic energy loss. Here, $E_{\text {ion }}$ and $E_{\text {exc }}$ are the ionization and excitation potentials, $K_{\text {exc }}$ and $K_{e l}$ are the excitation and elastic collision rate factors, and $V_{s}=\frac{1}{2} T_{e} \ln [M /(2 \pi m)]$ is the sheath potential, with $m$ the electron mass. By then using the known radial density factors $\left(\gamma_{9.5}\right.$ and $\left.\gamma_{17.5}\right)$, the thrust can be found using Eq. (7). The global model results (solid and dashed lines) are plotted in Figs. 2-3. Here, good agreement is seen with the measured electron temperatures, but only moderate agreement with the plasma density and thrust, with deviations of about $20 \%$ $40 \%$. The under predicted density (and hence thrust) is a consequence of the assumption of a uniform plasma density in the global model. In reality, this is not the case, and thus, the power loss to the wall is lower, which would result in a larger maximum density.

Two important performance measures for electric propulsion systems are the specific impulse, $I_{s p}=T /\left(\dot{m} g_{0}\right)$, and the thruster efficiency, $\eta_{T}=T^{2} /\left(2 \dot{m} P_{0}\right)$, where $T$ is the total system thrust, $g_{0}$ is the sea level gravitational acceleration, $\dot{m}$ is the total input propellant flow rate, and $P_{0}$ is the input power. Neglecting the thrust contribution from the neutral gas pressure and using the thrust measurements in Fig. 3, the present system has a maximum $I_{s p} \approx 230 \mathrm{sec}$ and $\eta_{T}<1 \%$, which is very poor compared with conventional electric propulsion systems. ${ }^{9}$ Part of the poor performance can be traced to a low propellant utilization, which gives a measure of how much of the input gas is ionized and is defined as $\eta_{d}=\dot{m}_{i} / \dot{m}$, where $\dot{m}_{i}$ is the total ion mass flow rate at the thruster exit. The ion mass flow rate can be approximately given by $\dot{m}_{i}=M n_{e} u_{B} A_{e}$, where $n_{e}$ is the density near the exit where the ions reach the Bohm velocity. The neutral flow rate at the exit can be given by $\dot{m}_{n}=1 / 4 M n_{g} v_{g} A_{e}$, where $v_{g}$ is the neutral gas velocity. Since the total mass flow rate is $\dot{m}=\dot{m}_{i}+\dot{m}_{n}$, the propellant utilization can be written as

$$
\eta_{d}=\frac{\dot{m}_{i}}{\dot{m}}=\frac{1}{1+\frac{n_{g} v_{g}}{4 n_{e} u_{B}}} .
$$

In the present study, $n_{g} \sim 10^{20} \mathrm{~m}^{-3}, n_{e} \sim 10^{18} \mathrm{~m}^{-3}$, and $u_{B} \sim 3500 \mathrm{~ms}^{-1}$, and thus, the propellant utilization is quite low at about $20 \%-30 \%$. An analysis of the global model results shows that most of the plasma/power loss in the system occurs at the radial boundaries (since the radial loss area is much larger than the axial boundaries). If the thruster cavity could be redesigned, improvements in performance would occur, and a larger propellant utilization could be obtained. A better option, however, would be to effectively eliminate radial losses altogether, which might be achievable with a correctly applied magnetic field. There is experimental evidence to suggest this approach is feasible ${ }^{2}$ and does indeed greatly improve performance.

The results from the present study (where no DL is present) show that the upstream electron pressure determines the thrust. In previous work (where a DL was present; Ref. 7), a similar result was obtained. This implies, as predicted in Ref. 12 , that the DL cannot directly increase the momentum of the plasma. It can only "convert" the electron pressure into ion momentum through the electric field in the DL; a process similar to what would occur in ambipolar type electric fields, and thus implies the DL does not perform a significant role.

In summary, we have shown analytically that the thrust from a collisionless plasma is given by the maximum upstream electron pressure, even in the presence of plasma divergence. Direct thrust measurements were performed on a plasma thruster for a range of operating conditions, and the results are in good agreement with the measured upstream electron pressure, verifying the analytical result.

The authors would like to thank Professor A. Fruchtman for many useful discussions. Dr. K. Takahashi would like to thank the Faculty of Engineering at Iwate University (sabbatical year), the TEPCO Research Foundation in Japan, the MEXT in Japan (Grant-in-Aid for Young Scientists A 22684031), and the SP3 laboratory (Australian Research Council Discovery Grant No. DP 1096653) for financial support.

${ }^{1}$ C. Charles, J. Phys. D: Appl. Phys. 42, 163001 (2009).

${ }^{2}$ O. V. Batishchev, IEEE Trans. Plasma Sci. 37, 1563 (2009).

${ }^{3}$ F. F. Chen, IEEE Trans. Plasma Sci. 36, 2095 (2008).

${ }^{4}$ J. Prager, T. Ziemba, R. Winglee, and B. R. Roberson, Phys. Plasmas 17, 013504 (2010).

${ }^{5}$ R. Winglee, T. Ziemba, L. Giersch, J. Prager, J. Carscaddenn, and B. R. Roberson, Phys. Plasmas 14, 063501 (2007).

${ }^{6}$ K. Takahashi, K. Oguni, H. Yamada, and T. Fujiwara, Phys. Plasmas 15, 084501 (2008)

${ }^{7}$ K. Takahashi, T. Lafleur, C. Charles, P. Alexander, R. W. Boswell, M. Perren, R. Laine, S. Pottinger, V. Lappas, T. Harles, and D. Lamprou, Appl. Phys. Lett. 98, 141503 (2011).

${ }^{8}$ S. Pottinger, V. Lappas, C. Charles, and R. W. Boswell, J. Phys. D: Appl. Phys. 44, 235201 (2011).

${ }^{9}$ D. M. Goebel and I. Katz, Fundamentals of Electric Propulsion: Ion and Hall Thrusters (John Wiley \&Sons, New Jersey, 2008).

${ }^{10}$ A. Fruchtman, IEEE Trans. Plasma Sci. 36, 403 (2008).

${ }^{11}$ A. Fruchtman, IEEE Trans. Plasma Sci. 39, 530 (2011).

${ }^{12}$ A. Fruchtman, Phys. Rev. Lett. 96, 065002 (2006).

${ }^{13}$ F. F. Chen, Introduction to Plasma Physics and Controlled Fusion Volume 1: Plasma Physics, 2nd ed. (Springer, New York, 2006) Chap. 7. pp. 238-239, Eqns. [7-41] and [7-42].

${ }^{14}$ M. D. West, C. Charles, and R. W. Boswell, J. Propul. Power 24, 134 (2008).

${ }^{15}$ T. E. Sheridan, Phys. Plasmas 7, 3084 (2000).

${ }^{16}$ T. Lafleur, C. Charles, and R. W. Boswell, J. Phys. D: Appl. Phys. 44, 055202 (2011)

${ }^{17}$ M. A. Lieberman and A. J. Lichtenberg, Principles of Plasma Discharges and Materials Processing, 2nd ed. (John Wiley \& Sons, New Jersey, 2005). 